\section{Modeling Tomorrow's Biorefinery-the NREL Biochemical Pilot Plant}

Turning great ideas into viable solutions requires the ability to test theories under real world conditions. Few companies have the resources to build pilot-scale processing plants to test their ideas. The U.S. Department of Energy's (DOE) National Renewable Energy Laboratory (NREL) helps by sharing its world-class equipment and expertise with industry and other research organizations through a variety of contractual arrangements.

At the NREL campus in Golden, Colo., researchers use state-of-the-art laboratories to develop and improve the technologies that convert biomass to fuels, chemicals, and materials. One of the most important tools available to biomass researchers is the Biochemical Pilot Plant housed in the Alternative Fuels User Facility (AFUF). In this facility, researchers test ideas for creating highvalue products from cellulosic biomass. They can test individual processing steps at the bench scale or they can test entire conversion processes at the "mini-pilot plant" scale (10- to 100-L vessel size) or at the pilot plant scale (one ton feedstock per day).

A model biochemical process for deriving usable products from biomass involves thermochemical pretreatment of the biomass, followed by enzymatic hydrolysis, fermentation, and product separation and recovery. The AFUF has equipment for performing all of these operations.

\section{Pretreatment}

After it is mechanically milled, biomass is "pretreated"- a process that typically uses heat, pressure, and chemical catalysts such as acids to break down hemicellulose into its component sugars (hydrolysis) and make the cellulosic fraction accessible

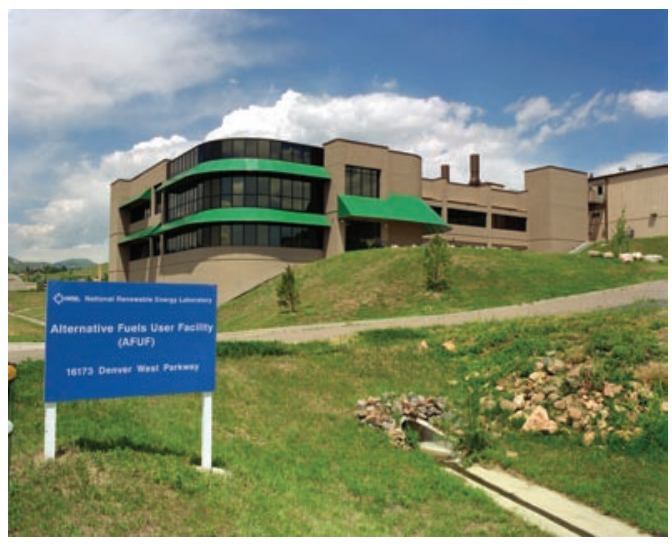

NREL's Alternative Fuels User Facility is an integral part of the National Bioenergy Center, which leads national laboratory research and development for the Biomass Program.

to enzymatic hydrolysis. NREL has historically focused on pretreatment processes that use dilute sulfuric acid; however, all of the pretreatment equipment can be used to explore alternative pretreatment chemistries.

\section{Pretreatment Equipment}

Bench-scale (less than 10-L working volume) pretreatment equipment:

- Batch 4-L steam gun

- Batch 1-L high solids stirred-tank reactor.

Pilot-scale systems:

- Continuous one ton biomass per day (dry basis) capacity vertical pressure reactor system

- Continuous $200 \mathrm{~kg}$ per day (dry basis) capacity horizontal-screw pressure reactor system

-130-L high solids steam-jacketed and steam-injected batch mixed reactor.
What Is a Biorefinery?

At a petroleum refinery, crude oil is processed into a variety of fuels and chemicals. DOE and NREL are leading efforts to develop a similar refinery model based on biomass. Biomass includes all plant and plant-derived material, but NREL research focuses particularly on cellulosic materials-the fibrous parts of plants, as opposed to the starch, sugar, and oil already used for food and fuel.

The three primary components of biomass are cellulose, hemicellulose, and lignin. The challenge is to economically break down the cellulose and hemicellulose into their component sugars-one way to do this is through a process known as biochemical biomass conversion. In a biorefinery, the component sugars are then processed into ethanol or other fuels, chemicals, or materials.

"I am pleased to note that some of the most promising recent breakthroughs and accomplishments in ethanol and biobased products

are being developed by

our Department's National Renewable Energy Laboratory."

Energy Secretary Samuel Bodman July 20, 2005 


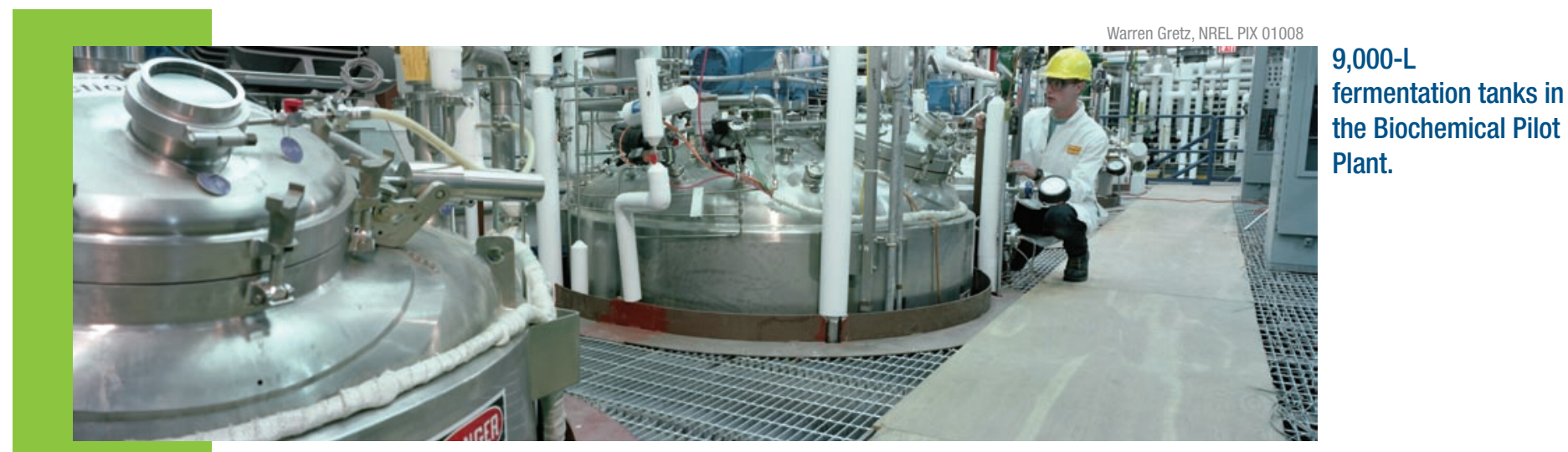

Enzymatic Hydrolysis

After pretreatment, the residual solids are treated with enzymes to break down the cellulose to sugar - a process known as enzymatic hydrolysis. NREL researchers have determined that enzymatic hydrolysis of cellulose has the greatest potential to achieve cost-effective biomass conversion.

\section{Hydrolysis Equipment}

NREL has a wide range of reactors for conducting experiments and assessing performance.

\section{Fermentation}

After hydrolysis, the sugars obtained from pretreatment and enzymatic hydrolysis are fermented to ethanol or otherwise processed to desired products. NREL has developed an improved bacterium (Zymomonas mobilis) capable of cofermenting both glucose (the six-carbon sugar derived from cellulose) and xylose (the primary sugar from hemicellulose, which is a five-carbon sugar that is more difficult to ferment). This patented strain holds promise for reducing capital equipment costs and streamlining process operations in a cellulose-based ethanol production plant. For a biorefinery, however, it may prove advantageous to ferment the sugar streams separately to produce different products. AFUF fermentation reactors can be used to test a variety of prospective aerobic or anaerobic bioprocesses in batch, fed-batch, or continuous operation.

\section{Fermentation Equipment}

Pilot-scale systems available in the AFUF include:

- A 20-L fermentor, two 160-L fermentors, two 1,500-L fermentors, and four 9,000-L fermentors
- Numerous sterilizable support and feed vessels ranging in size from 100 to $9,000 \mathrm{~L}$.

\section{Product Separation and Recovery}

By fermenting sugars we produce ethanol or other products that must then be effectively recovered. Biorefineries will probably rely on a variety of separation, purification, and recovery technologies. AFUF's fermentation and downstream separations and product recovery sections can also be operated for industrial partners who develop processes that start with sugars instead of raw biomass.

\section{Separation and Recovery Equipment}

Pilot-scale systems include systems for distillation/evaporation:

- $10-\mathrm{m}$ tall by $0.5-\mathrm{m}$ diameter sieve tray distillation column

- Continuous forced recirculation evaporator for concentrating or evaporative operations.

Pilot-scale systems for centrifugation include:

- Small solid bowl high-speed centrifuge $(17,000 \mathrm{G})$ for cell recovery

- Sterilizable continuous $100-\mathrm{L}$ per hour capacity high performance decanter $(5,000 \mathrm{G})$

- Continuous 1,200-L per hour capacity decanting centrifuge $(3,000 \mathrm{G})$

- A semi-automatic basket centrifuge (900 G) with automatic feed and discharge capability.

Pilot-scale systems for filtration include:

- Semi-continuous pressure belt filter

- Continuous ultrafiltration/reverse osmosis system.

Pilot-scale systems for purification include:

- A continuous ion exchange/chromatography moving bed system. 


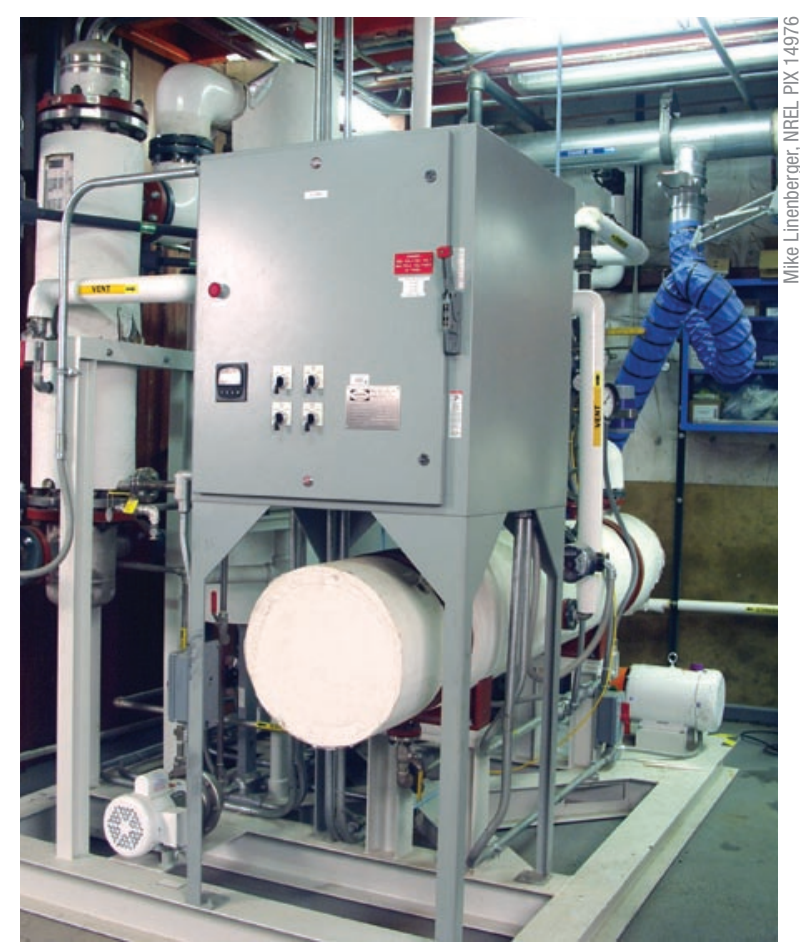

This evaporation system concentrates sugar-rich streams or removes volatile compounds.

\section{Process Integration}

Creating cost-effective industrial processes involves integrating the different bioconversion processing steps. NREL researchers are adept at integrating bioconversion processes and using the AFUF's systems to test prospective processes as they would be used in production. NREL researchers also have extensive experience supporting specific industrial partner research and development activities aimed at commercializing the next generation of bioconversion and bio-processing technologies.

\section{Compositional Analysis and Process Monitoring}

NREL researchers are well versed in biomass compositional analysis and process monitoring, and can reliably assess mass and element balance closures across discrete process steps. NREL analytical chemists have expertise in developing, applying, and refining analytical methods to determine the composition of a wide range of biomass feedstocks and process intermediates. Components monitored include polymeric and monomeric sugars, lignin, organic acids, protein, sugar degradation products, extractives, ash, and moisture.
Available tools include:

- A range of spectrometers (infrared, visible, ultraviolet), automated extraction units, and high performance liquid chromatographs equipped with a variety of columns and detector systems.

- "Rapid analysis" methods that use multivariate techniques to extract compositional information directly from complex sample spectra. Such methods enable accurate and low-cost determination of multiple components in solid, liquid, and even slurry samples, and are being developed to speed process development and to enable online compositional analysis during processing operations.

- A sophisticated data acquisition and control system that permits effective real-time process control and monitoring during any mode of operation (batch, fed-batch, or continuous).

- Dedicated mass spectrometers that permit on-line monitoring of fermentor exhaust gas compositions.

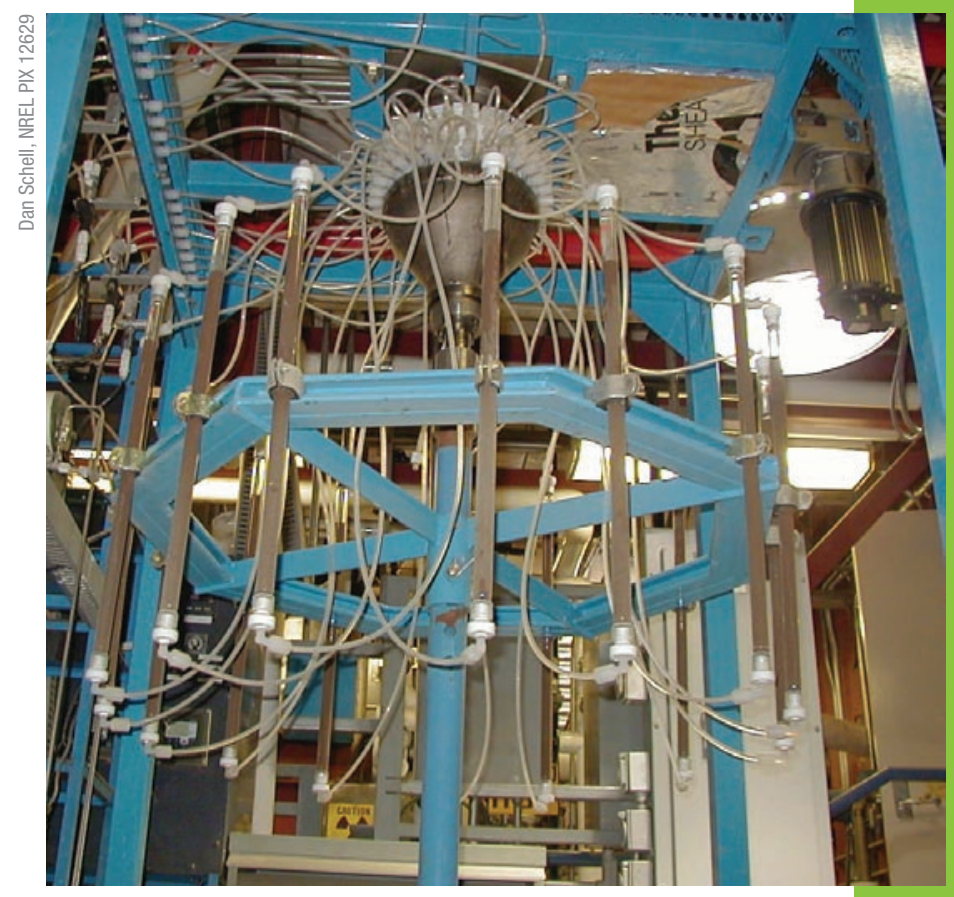

Researchers use this continuous ion exchange/ chromatography system for product recovery and purification. 


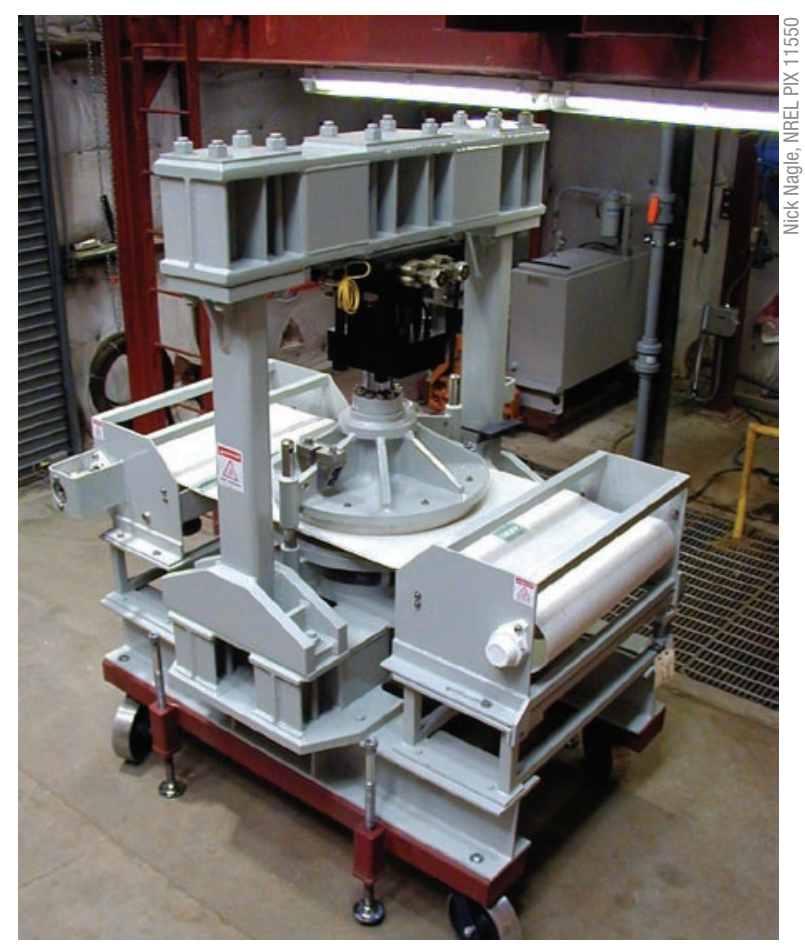

With this pressurized filter press system, researchers can separate liquid hydrolyzate from the remaining solids at high temperature using NREL's patented hot-wash process.

\section{Creating an Integrated Biorefinery Research Facility}

The AFUF has much of the equipment required to pilot biomass-to-ethanol processes. It does not, however, contain all the necessary equipment, nor is it large enough, to fully test integrated biorefinery operations. In 2009, DOE will add extensive processing, separations, and materials handling equipment that will allow us to test fully integrated biorefining processes and research alternative concepts and technologies.

For example, DOE plans to install frontend processing trains for testing different pretreatment chemistries. It also plans to add equipment to prepare residues from biochemical conversion for subsequent thermochemical conversion.

The expanded facility will provide a pilot-scale plant that is both large enough and flexible enough to accelerate biorefining process development and deployment. As different research activities are developed and tested, researchers and industrial partners will be able to add or remove equipment, operate multiple process trains, and reconfigure biomass conversion processes. Expanded analytical laboratory facilities will support biochemical process research and development and will allow us to more efficiently and accurately analyze samples throughout the process.

\section{For More Information}

For more information about NREL's AFUF, including inquiries about using the Biochemical Pilot Plant facilities, contact John Ashworth (303-384-6858,john_ashworth@nrel.gov), or visit our Web site at www.nrel.gov/biomass.

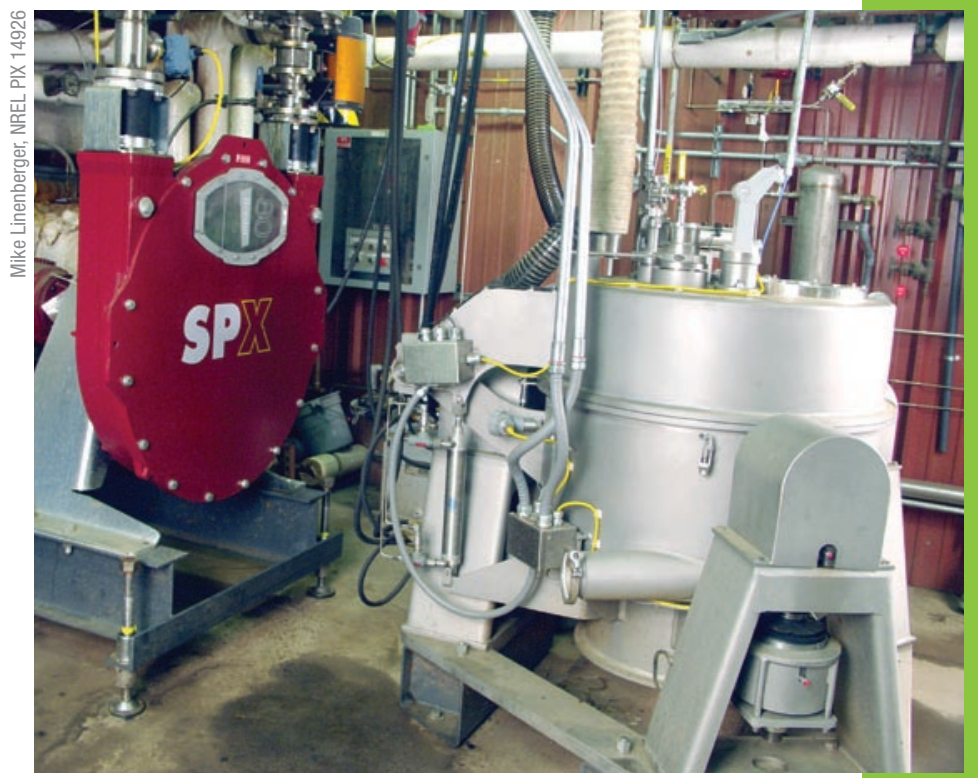

Automated basket centrifuge and pump separate sugarrich liquors from pretreated biomass slurries.

\section{A Strong Energy Portfolio for a Strong America}

Energy efficiency and clean, renewable energy will mean a stronger economy, a cleaner environment, and greater energy independence for America. Working with a wide array of state, community, industry, and university partners, the U.S. Department of Energy's Office of Energy Efficiency and Renewable Energy invests in a diverse portfolio of energy technologies.

For more information contact: EERE Information Center 1-877-EERE-INF (1-877-337-3463) www.eere.energy.gov
Produced for the U.S. Department of Energy by the National Renewable Energy Laboratory, a DOE national laboratory

DOE/GO-102008-2403, March 2008 